\title{
Diagnóstico de la capacidad para el ejercicio de las funciones esenciales de salud pública en países de Mesoamérica, los estados mexicanos de Chiapas y Quintana Roo y la República Dominicana
}

\author{
Miguel Ángel González Block, ${ }^{1}$ Luz María González Robledo \\ y Silvia Magali Cuadra Hernández ${ }^{1}$
}

Forma de citar

González Block MA, González Robledo LM, Cuadra Hernández SM. Diagnóstico de la capacidad para el ejercicio de las funciones esenciales de salud pública en países de Mesoamérica, los estados mexicanos de Chiapas y Quintana Roo y la República Dominicana. Rev Panam Salud Publica. 2013;33(4):271-9.

RESUMEN Objetivo. Caracterizar la capacidad para el desempeño de las funciones esenciales de la salud pública (FESP) de las instituciones públicas y privadas en países de Mesoamérica, los estados mexicanos de Chiapas y Quintana Roo y la República Dominicana.

Métodos. Se aplicó una encuesta en línea a 83 organizaciones de Belice, Costa Rica, El Salvador, Guatemala, Honduras, Nicaragua, Panamá, la República Dominicana y los estados mexicanos de Chiapas y Quintana Roo sobre la capacidad de cumplir cada una de las 11 FESP. Los resultados se validaron en un taller con representantes de los ministerios de salud de los siete países y los dos estados mexicanos participantes.

Resultados. La mayor capacidad para el desempeño se identificó en la FESP 1 (monitoreo, evaluación y análisis del estado de salud de la población), la FESP 2.1 .1 (vigilancia, investigación y control de riesgos y amenazas a la salud pública para enfermedades infecciosas) y la FESP 5 (desarrollo de políticas y planificación en salud). La mayor debilidad se encontró en la FESP 2.1.2 (vigilancia, investigación y monitoreo de las enfermedades no infecciosas). Las asimetrías en el desempeño de las FESP al interior de cada país indican debilidades en las funciones de los laboratorios y de la investigación en salud pública.

Conclusiones. Se requiere mejorar el desempeño estratégico en la mayor parte de las FESP en los países y territorios analizados y reforzar la infraestructura, el equipamiento y los recursos humanos, tanto a nivel estratégico como táctico. Se debe aplicar un enfoque regional para aprovechar la capacidad diferencial con vistas al fortalecimiento y el apoyo técnico cooperativo.

Palabras clave Sistemas de salud; funciones esenciales de la salud pública; América Central; México; República Dominicana.

Los países de América Central y los once estados del centro-sur de México

\footnotetext{
Centro de Investigación en Sistemas de Salud, Instituto Nacional de Salud Pública, Cuernavaca, Morelos, México.

2 Universidad Autónoma del Estado de Morelos, Cuernavaca, Morelos, México. La correspondencia se debe dirigir a Luz María González Robledo. Correo electrónico: luz.gonzalez@uaem.mx
}

conforman la subregión de Mesoamérica y junto a la República Dominicana conforman el Proyecto de Integración y Desarrollo de Mesoamérica y el Sistema Mesoamericano de Salud Pública. Los miembros de este proyecto, aunque diversos entre sí, comparten condiciones de salud, cultura y flujos poblacionales comunes, así como una larga historia de esfuerzos para el fortalecimiento de sus sistemas de salud (1). En conjunto, los participantes en este proyecto reúnen una población estimada en 88,6 millones de personas: 43,8 millones en los siete países centroamericanos, 10,1 en la República Dominicana y 34,7 millones radi- 
can en los once estados mesoamericanos de México. En general, estos territorios enfrentan problemas de salud emergentes con determinantes sociales y ambientales similares, a la vez que encaran el rezago epidemiológico —que se manifiesta en la coexistencia de enfermedades infecciosas y crónico-degenerativas y el envejecimiento de su población con altas tasas de natalidad, entre otras características-, que inciden en el aumento de las necesidades de salud. Sus sistemas sanitarios están constituidos, en la mayoría de los casos, por instituciones públicas y privadas focalizadas en grupos específicos de la población, mientras sus ministerios y secretarías de salud coordinan el sector y vigilan el cumplimiento del marco regulatorio. Esta organización segmentada de la salud pública genera inequidad e ineficiencia (1-4).

La herramienta de análisis y evaluación de las 11 funciones esenciales de la salud pública (FESP) propuesta por la Organización Panamericana de la Salud (OPS) ha demostrado ser útil para formular acciones de fortalecimiento e identificar capacidades para enfrentar riesgos y amenazas a la salud (5-7). La Asociación Internacional de Institutos Nacionales de Salud (IANPHI) utilizó esta herramienta modificada para realizar una evaluación de las FESP en los institutos nacionales que forman parte de su red $(8,9)$.

El objetivo del presente trabajo fue caracterizar la percepción de los funcionarios de las instituciones públicas y privadas en países de Mesoamérica, los estados mexicanos de Chiapas y Quintana Roo y la República Dominicana sobre la capacidad para el desempeño de las FESP a niveles institucional, local y general del conjunto de territorios estudiados.

\section{MATERIALES Y MÉTODOS}

Se realizó un diagnóstico basado en la percepción de funcionarios clave sobre la capacidad para el desempeño de las FESP en organizaciones de los sistemas de salud de Belice, Costa Rica, El Salvador, Guatemala, Honduras, Nicaragua, Panamá, República Dominicana y los estados mexicanos de Chiapas y Quintana Roo por ser fronterizos con los países centroamericanos y compartir muchas de sus características distintivas. En este diagnóstico participaron como instituciones acompañantes el Instituto Costarricense de Investigación y Enseñanza en
Nutrición y Salud (INCIENSA), de Costa Rica, y el Instituto Conmemorativo Gorgas de Estudios de la Salud, de Panamá.

Se utilizó la metodología de la OPS (5) adaptada para desagregar la FESP 2 (vigilancia de salud pública, investigación y control de riesgos y daños en salud pública) en cinco subfunciones, por su alta prioridad para el Sistema Mesoamericano de Salud Pública. La relación detallada de cada FESP se presenta en el cuadro 1. Para registrar el desempeño en estos temas se utilizó el instrumento de la IANPHI (8), que emplea una escala con ejemplos hipotéticos para estandarizar las escalas de medición.

Se identificaron 171 organizaciones académicas y de servicios, tanto del ámbito gubernamental como no gubernamental y privado, que gozan de reconocimiento por su desempeño en al menos una de las FESP en los territorios estudiados. La identificación se hizo inicialmente a partir de la información contenida en los directorios del Instituto Nacional de Salud Pública, de México, y las recomendaciones del Instituto Gorgas y el INCIENSA; la relación inicial se amplió posteriormente con otras instituciones mediante la técnica de "la bola de nieve".

Después de presentar el proyecto de evaluación a los representantes de las organizaciones seleccionadas, mediante correos electrónicos y un sitio en Internet, se realizó un seguimiento telefónico para facilitar el proceso de completamiento de la encuesta que debían responder. Finalmente, la muestra de estudio quedó compuesta por representantes de todos los ministerios de salud pública, organizaciones de la sociedad civil e instituciones privadas, que voluntariamente completaron la encuesta. En su conjunto, las instituciones y organizaciones participantes reflejaban todas las vertientes que componen los sistemas de salud de los países centroamericanos, la República Dominicana y los estados mexicanos participantes, donde por su fragmentación, las FESP no son competencia absoluta de las instituciones gubernamentales. Aunque se trata de una muestra de conveniencia que no puede considerarse representativa de las organizaciones civiles y privadas de los países y estados mexicanos participantes, la inclusión de todos los ministerios y secretarías de salud aporta una adecuada robustez a los resultados y permite llegar a una primera exploración en el campo de las organizaciones relacionadas con la salud pública y las FESP en este conjunto de territorios.

La encuesta se aplicó a representantes organizacionales mediante una cédula en línea (10), disponible desde noviembre de 2008 hasta marzo de 2009. La capacidad de cumplir cada una de las 11 FESP evaluadas se midió a partir de la percepción del desempeño de entre 3 y 10 competencias, para un total de 86 competencias. Cada competencia se clasificó mediante una escala de cuatro opciones (no se aborda, se aborda algo, se aborda mucho, se aborda integralmente). Con el fin de medir la capacidad, tanto a nivel institucional como local y general (del conjunto de territorios estudiados), el análisis de las FESP se realizó en cuatro dimensiones: a) para cada FESP y para el conjunto de las FESP en general; b) para las competencias abordadas por cada institución (capacidad táctica, que refleja las competencias abordadas en cada FESP por cada institución, sin promediar las competencias no abordadas); c) para el conjunto de las instituciones de cada país o estado analizado (capacidad estratégica, que abarca todas las competencias necesarias para el abordaje integral de cada FESP y al promediar ajusta a cero los valores de las competencias no abordadas); y d) para el desempeño tanto a nivel local como general.

Para calificar los desempeños táctico y estratégico de cada FESP, se empleó el promedio de los valores de sus competencias en una escala de 1 a 10 (se consideró la capacidad como limitada cuando el valor promedio era de 0 a 3,9; específica si era de 4,0-5,9; amplia, de 6,0-7,9; e integral, de 8,0-10,0). Para la medición local, se promediaron los valores de las instituciones de cada país o estado mexicano participante, mientras que para obtener el promedio general se tomaron todas las instituciones de los territorios analizados.

Para validar los resultados y consensuar las recomendaciones, se realizó un taller en San José, Costa Rica, en el que participaron al menos dos representantes de los ministerios de salud de los siete países y los dos estados mexicanos participantes (22 en total). Antes del taller, los participantes pudieron revisar el informe y consultarlo con investigadores y funcionarios locales. En el taller se identificaron fortalezas, oportunidades, debilidades y amenazas para el 


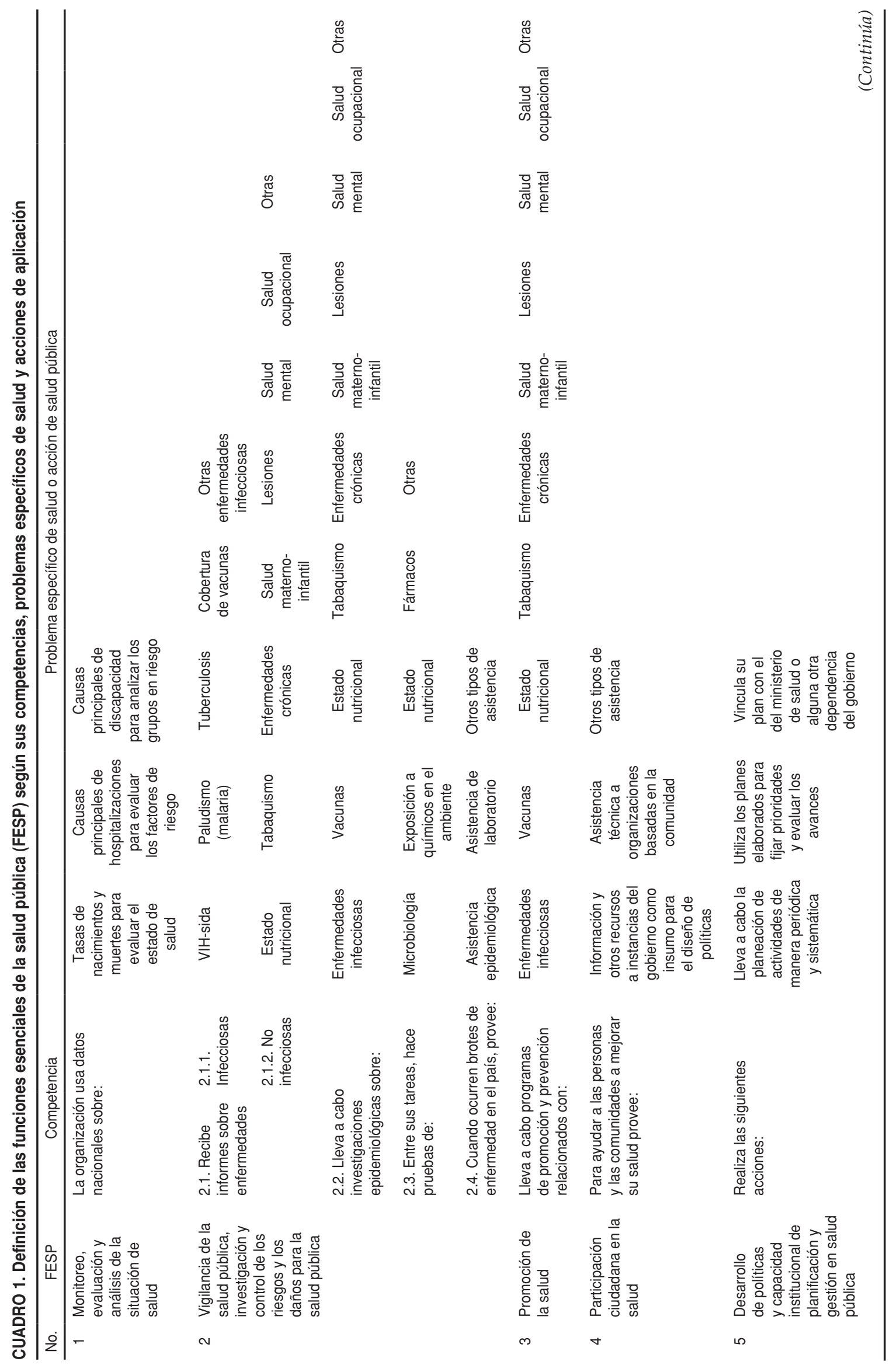




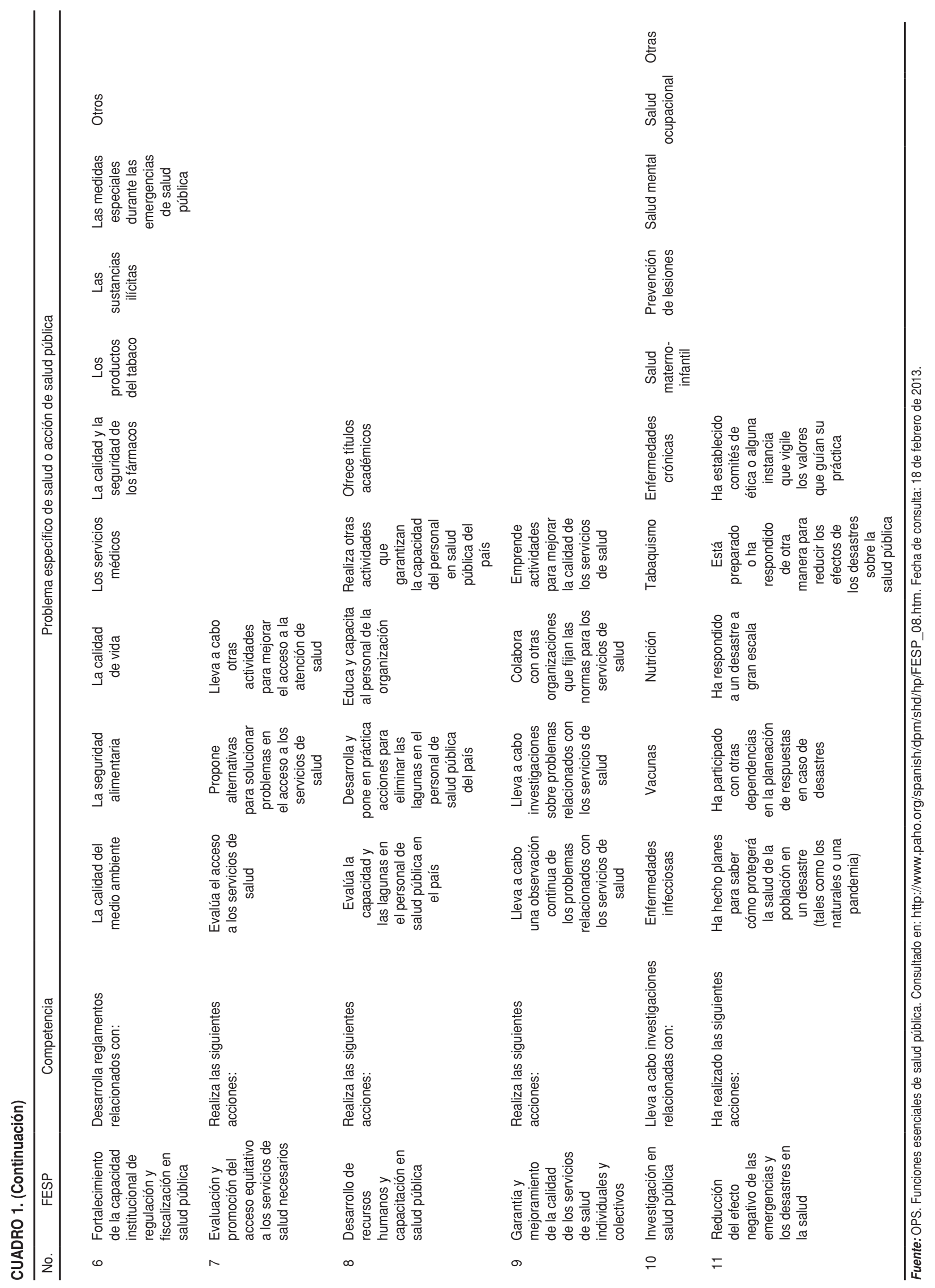


desempeño de las FESP mediante cuatro guías de discusión y ponderación en pequeños grupos por país. Estas guías, elaboradas y probadas previamente por los investigadores, contenían anexos con ejemplos que ayudaban a enfocar el análisis en una amplia gama de factores. Después de validar el diagnóstico situacional a partir de los resultados para cada FESP y las fortalezas y oportunidades identificadas para cada una de ellas en cada país y estado mexicano, se propusieron acciones de fortalecimiento, tanto a nivel nacional como para el conjunto de territorios analizados. Con esta información, se procedió a identificar cinco oportunidades y cinco amenazas, según el contexto externo, de cada acción recomendada. Cada aspecto recibió una calificación y una ponderación relativa que permitieron llegar a una calificación integral para definir la factibilidad de que la red de organizaciones de salud pública pudiera abordar las FESP, según su contexto externo. Se utilizó el mismo procedimiento para analizar, en el contexto interno, las fortalezas y las debilidades y determinar la calificación general de cada acción que, finalmente, reflejaba hasta qué grado las condiciones eran favorables para la acción. El ejercicio culminó con la elaboración de propuestas de acción y colaboración técnica.

El proyecto recibió la aprobación de la Comisión de Ética e Investigación del Instituto Nacional de Salud Pública, de México.

\section{RESULTADOS}

Se obtuvo respuesta de $83(48,5 \%)$ de las organizaciones invitadas; de ellas, 51 $(61,4 \%)$ eran públicas, principalmente de los ministerios de salud o proveedores de servicios; $31(37,4 \%)$ privadas, princi-

CUADRO 2. Instituciones participantes que respondieron la encuesta en línea, según el país y tipo de institución

\begin{tabular}{|c|c|c|c|c|c|c|}
\hline \multirow[b]{3}{*}{ País/estado mexicano } & \multirow{3}{*}{$\begin{array}{l}\text { Instituciones } \\
\text { invitadas }\end{array}$} & \multicolumn{4}{|c|}{ Instituciones participantes } & \multirow{3}{*}{$\begin{array}{c}\text { Tasa de respuesta } \\
(\%)\end{array}$} \\
\hline & & \multirow[b]{2}{*}{ Total } & \multicolumn{3}{|c|}{ Tipo de institución } & \\
\hline & & & Pública & Privada & Mixta & \\
\hline Belice & 19 & 5 & 5 & 0 & 0 & 26,3 \\
\hline Costa Rica & 17 & 13 & 10 & 3 & 0 & 76,5 \\
\hline El Salvador & 14 & 8 & 4 & 4 & 0 & 57,1 \\
\hline Guatemala & 17 & 6 & 2 & 3 & 1 & 35,3 \\
\hline Honduras & 10 & 6 & 3 & 3 & 0 & 60,0 \\
\hline México: Chiapas & 23 & 10 & 5 & 5 & 0 & 43,4 \\
\hline México: Quintana Roo & 30 & 13 & 7 & 6 & 0 & 43,3 \\
\hline Nicaragua & 15 & 10 & 4 & 6 & 0 & 66,7 \\
\hline Panamá & 14 & 8 & 8 & 0 & 0 & 57,1 \\
\hline República Dominicana & 12 & 4 & 3 & 1 & 0 & 33,3 \\
\hline Total & 171 & 83 & 51 & 31 & 1 & 48,5 \\
\hline
\end{tabular}

palmente académicas o proveedoras de servicios; y $1(1,2 \%)$ era mixta, pues recibía fondos públicos y privados (cuadro 2). Las instituciones académicas tuvieron la mayor representatividad en la muestra (53\% del total).

Las FESP de las instituciones participantes se agruparon por territorio; de ellos, el más representado fue Costa Rica, con 13 instituciones (tasa de respuesta de 76,5\%), y el menos representado fue la República Dominicana, con 4 (tasa de respuesta de 33,3\%).

En general, la mayor capacidad táctica se encontró en la FESP 2.1.1 (información sobre enfermedades infecciosas) y la FESP 5 (desarrollo de políticas, planificación y gestión) (cuadro 3).

Al analizar las capacidades estratégicas generales de las instituciones de los países o estados mexicanos participantes (en ambos casos a partir de los promedios), se encontró que las acciones de salud pública con mayor capacidad en el conjunto de instituciones analizadas fueron la FESP 5 (desarrollo de políticas, planificación y gestión) con 4,8 puntos, y la FESP 1 (monitoreo, evaluación y análisis) y la FESP 2.1.1 (información sobre enfermedades infecciosas) con 3,8 puntos (cuadro 4). Estos resultados demuestran que se han desarrollado las mayores capacidades para responder al perfil epidemiológico de la población. Pese a que la mayoría de los países presentan patrones de mortalidad con predominio de enfermedades crónico-degenerativas, muy pocos han desarrollado la FESP 2.1.2 (información sobre enfermedades no infecciosas), que alcanzó solamente 2,5 puntos. Las FESP con menor capacidad estratégica fueron la FESP 10 (investigación), con 1,6 puntos, y la FESP 2.3 (laboratorios), con 1,8 puntos (cuadro 4 ).

\section{Diagnósticos específicos}

$\mathrm{Al}$ analizar las capacidades tácticas (cuadro 3) y estratégicas (cuadro 4) de las instituciones de los países o estados mexicanos participantes se encontró lo siguiente:

Belice. La mayor capacidad táctica se observó en la FESP 2.1.1 (información sobre

CUADRO 3. Desempeño táctico en las funciones esenciales de la salud pública (FESP) de las redes de instituciones por país o estado mexicano

\begin{tabular}{|c|c|c|c|c|c|c|c|c|c|c|c|c|c|c|c|c|c|}
\hline \multirow[b]{2}{*}{ País/estado mexicano } & \multicolumn{16}{|c|}{ FESPa } & \multirow{2}{*}{$\begin{array}{l}\text { Todas las } \\
\text { FESPb }\end{array}$} \\
\hline & 1 & 2.1 .1 & 2.1 .2 & 2.2 & 2.3 & 2.4 & 2 & 3 & 4 & 5 & 6 & 7 & 8 & 9 & 10 & 11 & \\
\hline Belice & 7,4 & 8,1 & 6,0 & 5,0 & 4,0 & 5,6 & 5,7 & 4,8 & 4,9 & 7,2 & 5,7 & 6,0 & 4,6 & 5,5 & 4,0 & 5,8 & 5,3 \\
\hline Costa Rica & 6,0 & 6,9 & 5,5 & 4,9 & 6,5 & 6,8 & 6,1 & 6,0 & 6,2 & 6,3 & 6,3 & 5,2 & 5,9 & 4,9 & 5,2 & 6,0 & 5,0 \\
\hline El Salvador & 5,9 & 6,6 & 4,8 & 5,8 & 4,9 & 6,1 & 5,7 & 6,2 & 5,7 & 6,5 & 5,6 & 5,4 & 4,6 & 4,7 & 4,7 & 6,3 & 5,0 \\
\hline Guatemala & 5,3 & 6,8 & 4,5 & 6,1 & 6,1 & 8,4 & 6,4 & 5,6 & 5,2 & 6,5 & 4,9 & 4,3 & 6,0 & 4,5 & 5,8 & 5,5 & 5,2 \\
\hline Honduras & 7,3 & 6,8 & 5,8 & 5,8 & 5,9 & 5,8 & 6,0 & 6,2 & 6,2 & 7,7 & 5,2 & 8,0 & 6,2 & 5,0 & 6,0 & 6,3 & 5,8 \\
\hline México: Chiapas & 6,9 & 6,3 & 5,3 & 5,9 & 5,7 & 6,0 & 5,8 & 5,3 & 8,6 & 7,1 & 6,2 & 6,0 & 5,4 & 5,9 & 4,5 & 6,0 & 5,6 \\
\hline México: Quintana Roo & 6,0 & 6,0 & 5,0 & 4,8 & 5,1 & 6,5 & 5,5 & 6,0 & 5,0 & 6,4 & 5,7 & 6,9 & 6,0 & 4,9 & 4,7 & 5,7 & 5,0 \\
\hline Nicaragua & 5,5 & 6,4 & 5,4 & 5,4 & 4,9 & 4,4 & 5,3 & 5,8 & 6,8 & 6,1 & 4,9 & 4,8 & 5,6 & 4,6 & 4,5 & 5,6 & 4,9 \\
\hline Panamá & 5,7 & 8,6 & 6,7 & 7,8 & 7,9 & 6,4 & 7,5 & 5,6 & 5,8 & 6,7 & 6,5 & 7,8 & 6,4 & 6,2 & 5,2 & 4,8 & 5,7 \\
\hline República Dominicana & 4,6 & 5,3 & 5,0 & 5,0 & 4,0 & 4,0 & 4,7 & 5,0 & 5,5 & 5,3 & 4,0 & 5,0 & 5,3 & 5,3 & 4,8 & 4,0 & 4,5 \\
\hline
\end{tabular}

\footnotetext{
a La relación de las FESP se puede consultar en el cuadro 1.

b Promedio de la capacidad táctica para cada FESP (sin considerar subfunciones de la FESP 2).
} 
CUADRO 4. Desempeño estratégico en las funciones esenciales de la salud pública (FESP) de las redes de instituciones por país o estado mexicano

\begin{tabular}{|c|c|c|c|c|c|c|c|c|c|c|c|c|c|c|c|c|c|}
\hline \multirow[b]{2}{*}{ País/estado mexicano } & \multicolumn{16}{|c|}{ FESPa $^{a}$} & \multirow{2}{*}{$\begin{array}{c}\text { Todas las } \\
\text { FESPb }\end{array}$} \\
\hline & 1 & 2.1 .1 & 2.1 .2 & 2.2 & 2.3 & 2.4 & 2 & 3 & 4 & 5 & 6 & 7 & 8 & 9 & 10 & 11 & \\
\hline Belice & 5,4 & 7,5 & 4,4 & 1,8 & 1,1 & 4,3 & 3,8 & 3,8 & 3,9 & 6,4 & 2,6 & 5,1 & 2,9 & 3,8 & 1,1 & 4,1 & 3,6 \\
\hline Costa Rica & 2,9 & 2,9 & 1,8 & 1,3 & 2,5 & 2,7 & 2,2 & 1,9 & 3,5 & 5,1 & 1,9 & 1,6 & 2,8 & 3,0 & 1,4 & 1,3 & 2,3 \\
\hline El Salvador & 3,9 & 4,4 & 1,9 & 2,7 & 2,2 & 1,3 & 2,5 & 2,9 & 1,3 & 1,3 & 2,1 & 1,3 & 2,3 & 2,9 & 1,7 & 3,8 & 2,1 \\
\hline Guatemala & 4,6 & 4,7 & 2,5 & 2,8 & 2,7 & 5,0 & 3,5 & 2,2 & 3,2 & 5,9 & 2,2 & 2,7 & 4,3 & 3,2 & 1,5 & 2,7 & 3,2 \\
\hline Honduras & 6,2 & 5,4 & 2,9 & 2,6 & 2,5 & 3,2 & 3,3 & 3,0 & 3,4 & 5,3 & 3,8 & 4,8 & 3,0 & 2,6 & 3,5 & 4,3 & 3,5 \\
\hline México: Chiapas & 4,7 & 4,3 & 3,4 & 3,4 & 2,0 & 3,3 & 3,3 & 3,6 & 6,8 & 7,1 & 3,0 & 5,6 & 3,7 & 5,6 & 2,2 & 3,6 & 4,2 \\
\hline México: Quintana Roo & 3,4 & 2,2 & 2,4 & 1,5 & 1,4 & 1,8 & 1,8 & 3,7 & 2,4 & 4,8 & 1,2 & 3,7 & 1,2 & 2,1 & 1,0 & 3,0 & 2,3 \\
\hline Nicaragua & 3,8 & 4,5 & 3,0 & 2,6 & 0,9 & 1,7 & 2,5 & 2,4 & 4,3 & 5,7 & 0,8 & 3,3 & 3,0 & 3,6 & 1,7 & 3,5 & 2,8 \\
\hline Panamá & 3,3 & 4,5 & 3,5 & 3,0 & 3,4 & 2,8 & 3,4 & 2,3 & 2,9 & 4,8 & 3,6 & 3,8 & 2,7 & 3,3 & 1,1 & 1,7 & 3,0 \\
\hline República Dominicana & 3,4 & 2,3 & 2,0 & 1,2 & 0,8 & 0,3 & 1,3 & 1,0 & 3,2 & 3,2 & 1,1 & 4,0 & 2,6 & 3,5 & 2,0 & 1,5 & 2,1 \\
\hline Desempeño general & 3,8 & 3,8 & 2,5 & 2,1 & 1,8 & 2,5 & 2,5 & 2,6 & 3,2 & 4,8 & 2,0 & 3,3 & 2,6 & 3,1 & 1,6 & 2,8 & 2,6 \\
\hline
\end{tabular}

a La relación de las FESP se puede consultar en el cuadro 1.

b Promedio de la capacidad táctica para cada FESP (sin considerar subfunciones de la FESP 2).

enfermedades infecciosas), la FESP 1 (monitoreo, evaluación y análisis) y la FESP 5 (desarrollo de políticas, planificación y gestión). Las FESP con menor calificación fueron la FESP 2.3 (laboratorios), la FESP 10 (investigación) y la FESP 8 (desarrollo y capacitación de recursos humanos). Al analizar la capacidad para el desempeño estratégico, las FESP con mayor calificación fueron la FESP 2.1.1 (información sobre enfermedades infecciosas) y la FESP 5 (desarrollo de políticas, planificación y gestión) con una calificación de 6,4 puntos, lo que indica que existe una amplia capacidad para fijar prioridades, realizar planeaciones periódicas y sistemáticas de actividades - sobre todo en el área de enfermedades infecciosas- y vincularlas con los planes del ministerio de salud o de alguna dependencia gubernamental. Resalta la baja capacidad estratégica observada en la FESP 2.3 (laboratorios) y la FESP 10 (investigación).

Costa Rica. La mayor capacidad táctica encontrada fue en la FESP 2.1.1 (información sobre enfermedades infecciosas), la FESP 2.4 (asistencia ante brotes), la FESP 2.3 (laboratorios), la FESP 5 (desarrollo de políticas, planificación y gestión) y la FESP 6 (fortalecimiento de la regulación y la fiscalización). Las de menor capacidad táctica fueron la FESP 2.2 (investigaciones epidemiológicas), la FESP 9 (garantía y mejoramiento de la calidad de los servicios), la FESP 7 (evaluación y promoción del acceso equitativo a los servicios), la FESP 10 (investigación) y la FESP 2.1.2 (información sobre enfermedades no infecciosas). La capacidad para el desempeño táctico indica que existen las condiciones para la vigilancia epidemiológica de las enfermedades infecciosas y el control de brotes, apoyada por un sistema nacional de laboratorios. Asimismo, refleja mejoras en la elaboración de planes alineados con las políticas gubernamentales y en el cumplimiento de la regulación sanitaria. Respecto a la capacidad para el desempeño estratégico, la FESP 5 (desarrollo de políticas, planificación y gestión) tuvo la mayor calificación (5,1 puntos), lo que refleja una capacidad específica para fijar prioridades, realizar planeaciones periódicas y sistemáticas de actividades y vincularlas con los planes del ministerio de salud o de alguna dependencia gubernamental.

El Salvador. Las FESP con mayor capacidad táctica fueron la FESP 2.1.1 (información sobre enfermedades infecciosas), la FESP 5 (desarrollo de políticas, planificación y gestión), la FESP 11 (reducción del efecto negativo de emergencias y desastres), la FESP 3 (promoción de la salud) y la FESP 2.4 (asistencia ante brotes). La menor capacidad táctica se encontró en la FESP 8 (desarrollo y capacitación de recursos humanos), la FESP 9 (garantía y mejoramiento de la calidad de los servicios), la FESP 10 (investigación) y la FESP 2.1.2 (información sobre enfermedades no infecciosas). El Salvador cuenta con un elevado desempeño táctico, aunque todavía presenta debilidades en las acciones que ha abordado. En la capacidad estratégica, la FESP 2.2.1 (información sobre enfermedades infecciosas) tiene el mejor desempeño, con 4,4 puntos, que se acerca al nivel de capacidad específica. Se observaron avances en el uso de datos nacionales para evaluar el estado de salud de la población y establecer los perfiles de riesgo y las principales causas de hospitalización. La menor capacidad estratégica se encontró en la FESP 2.4 (asistencia ante brotes), la FESP 4 (participación ciudadana), la FESP 5 (desarrollo de políticas y capacidad institucional de planificación y gestión) y la FESP 7 (evaluación y promoción del acceso equitativo a los servicios).
Guatemala. La mayor capacidad táctica se concentró en la FESP 2.4 (asistencia ante brotes) y la FESP 2.1.1 (información sobre enfermedades infecciosas). La menor capacidad táctica se detectó en la FESP 7 (evaluación y promoción del acceso equitativo a los servicios), la FESP 2.1.2 (información sobre enfermedades no infecciosas), la FESP 9 (garantía y mejoramiento de la calidad de los servicios) y la FESP 6 (fortalecimiento de la regulación y la fiscalización). Las puntuaciones alcanzadas indican que las instituciones participantes cuentan con capacidad táctica, aunque todavía insuficiente. El mayor desempeño estratégico se encontró en la FESP 5 (desarrollo de políticas, planificación y gestión), con 5,9 puntos, lo que refleja una capacidad específica para fijar prioridades, realizar la planeación periódica de actividades y vincularlas con los planes del ministerio de salud o de alguna dependencia gubernamental. La FESP 10 (investigación) presentó la menor capacidad para el desempeño estratégico.

Honduras. La mayor capacidad táctica se encontró en la FESP 7 (evaluación y promoción del acceso equitativo a los servicios), la FESP 5 (desarrollo de políticas, planificación y gestión), la FESP 1 (monitoreo, evaluación y análisis) y la FESP 2.1.1 (información sobre enfermedades infecciosas). Las FESP con menor desempeño táctico fueron la FESP 9 (garantía y mejoramiento de la calidad de los servicios), la FESP 6 (fortalecimiento de la regulación y la fiscalización), la FESP 2.1.2 (información sobre enfermedades no infecciosas), la FESP 2.2 (investigaciones epidemiológicas) y la FESP 2.4 (asistencia ante brotes). La FESP con mayor capacidad para el desempeño estratégico fue la FESP 1 (monitoreo, evaluación y análisis), con 6,2 puntos, lo que refleja una capacidad amplia para usar datos nacionales para monitorear 
el estado de salud de la población, así como los principales factores de riesgo a los que está expuesta. La FESP con menor capacidad estratégica fue la FESP 2.3 (laboratorios), con 2,5 puntos.

México, Chiapas. Las FESP con mayor capacidad táctica fueron la FESP 4 (participación ciudadana), la FESP 5 (desarrollo de políticas, planificación y gestión), la FESP 1 (monitoreo, evaluación y análisis), la FESP 2.1.1 (información sobre enfermedades infecciosas) y la FESP 6 (fortalecimiento de la regulación y la fiscalización). Los principales retos se encontraron en la FESP 10 (investigación), la FESP 2.1.2 (información sobre enfermedades no infecciosas) y la FESP 3 (promoción de la salud). El desempeño táctico del conjunto de las instituciones reflejó una considerable capacidad para las acciones que deciden abordar. La FESP 5 (desarrollo de políticas, planificación y gestión) mostró mayor capacidad para el desempeño estratégico, con 7,1 puntos; esto indica una amplia capacidad para fijar prioridades, realizar la planeación sistemática de actividades y vincularlas con los planes del ministerio de salud o de alguna dependencia gubernamental. La FESP 2.3 (laboratorios) fue la de menor capacidad estratégica, con 2,0 puntos.

México, Quintana Roo. La mayor capacidad táctica se encontró en la FESP 7 (evaluación y promoción del acceso equitativo a los servicios) y la FESP 2.4 (asistencia ante brotes) y el menor desempeño táctico en la FESP 10 (investigación), la FESP 2.2 (investigaciones epidemiológicas), la FESP 9 (garantía y mejoramiento de la calidad de los servicios), la FESP 2.1.2 (información sobre enfermedades no infecciosa) y la FESP 4 (participación ciudadana). La FESP 5 (desarrollo de políticas, planificación y gestión) presentó mayor desempeño estratégico $(4,8$ puntos), lo que refleja la capacidad para fijar prioridades, realizar la planeación periódica y sistemática de actividades y vincularlas con los planes del ministerio de salud o de alguna dependencia gubernamental. La FESP 10 (investigación), la FESP 6 (fortalecimiento de la regulación y la fiscalización) y la FESP 8 (desarrollo y capacitación de recursos humanos) mostraron menor capacidad estratégica.

Nicaragua. La mayor capacidad táctica se observó en la FESP 4 (participación ciudadana), la FESP 2.1.1 (información sobre enfermedades infecciosas), la FESP 5 (desarrollo de políticas, planificación y gestión) y la FESP 3 (promoción de la salud). Estos resultados indican que hay una mayor capacidad en las áreas relacionadas con el perfil epidemiológico predominante en el país. La menor calificación la recibieron la FESP 2.4 (asistencia ante brotes), la FESP 10 (investigación), la FESP 9 (garantía y mejoramiento de la calidad de los servicios), la FESP 7 (evaluación y promoción del acceso equitativo a los servicios), la FESP 6 (fortalecimiento de la regulación y la fiscalización) y la FESP 2.3 (laboratorios). La FESP 5 (desarrollo de políticas, planificación y gestión) mostró mayor capacidad para el desempeño estratégico (5,7 puntos), lo que indica que se cuenta con capacidad para fijar prioridades, realizar la planeación periódica y sistemática de actividades y vincularlas con los planes del ministerio de salud o de alguna dependencia gubernamental. Las FESP con menor capacidad estratégica fueron la FESP 6 (fortalecimiento de la regulación y la fiscalización) y la FESP 2.3 (laboratorios).

Panamá. La mayor capacidad táctica se evidenció en la FESP 2.1.1 (información sobre enfermedades infecciosas), la FESP 2.3 (laboratorios), la FESP 2.2 (investigaciones epidemiológicas) y la FESP 7 (evaluación y promoción del acceso equitativo a los servicios). Los mayores retos se concentraron en la FESP 11 (reducción del efecto negativo de emergencias y desastres), la FESP 10 (investigación), la FESP 3 (promoción de la salud) y la FESP 1 (monitoreo, evaluación y análisis). La FESP 5 (desarrollo de políticas, planificación y gestión) mostró el mayor desempeño estratégico (4,8 puntos), lo que refleja la capacidad del país para fijar prioridades, realizar planeación periódica y sistemática de actividades y vincularlas con los planes del ministerio de salud o de alguna dependencia gubernamental. La FESP 10 (investigación) mostró la menor capacidad estratégica (1,1 puntos).

República Dominicana. Se observaron avances en la capacidad táctica para la FESP 4 (participación ciudadana), la FESP 2.1.1 (información sobre enfermedades infecciosas), la FESP 5 (desarrollo de políticas, planificación y gestión), la FESP 8 (desarrollo y capacitación de recursos humanos) y la FESP 9 (garantía y mejoramiento de la calidad de los servicios). Las menores calificaciones se encontraron en la FESP 2.3 (laboratorios), la FESP 2.4 (asistencia ante brotes), la FESP 6 (fortalecimiento de la regulación y la fiscalización), la FESP 11 (reducción del efecto negativo de emergencias y desastres) y la FESP 1 (monitoreo, evaluación y análi- sis). En cuanto a las capacidades estratégicas, la FESP 7 (evaluación y promoción del acceso equitativo a los servicios) mostró la mayor puntuación, lo que refleja una capacidad específica para evaluar el acceso de la población a los servicios de salud y promover acciones de mejoramiento en esta área. Este resultado podría estar asociado con el proceso de reforma del sistema de salud que ha emprendido este país en los últimos años. La República Dominicana muestra, en general, muy baja capacidad estratégica para todas las FESP.

\section{Planeación estratégica}

El ejercicio de planificación estratégica identificó como principales fortalezas de las instituciones de todos los países y estados mexicanos estudiados la disponibilidad de recursos humanos capacitados en la subregión, la capacidad para formar recursos humanos en el área, la existencia de tecnologías de información necesarios para esta capacitación y el cúmulo de buenas prácticas y experiencias en materia de salud pública.

Las principales debilidades identificadas en los países y estados mexicanos analizados fueron la falta de políticas y acciones de planificación de los recursos humanos para la salud y la falta de suficientes evaluaciones de su desempeño. En cuanto a los recursos materiales y técnicos, se encontraron deficiencias en la mayoría de los sistemas de información y divulgación, que dificultaban la priorización, así como el cumplimiento parcial de los planes estratégicos, la aún insuficiente toma de decisiones basadas en resultados científicos, una gestión institucional sujeta a cambios políticos y un limitado acceso a las nuevas tecnologías de la comunicación y la información.

Las oportunidades en el contexto subregional se concentran en el apoyo político entre los países, la cooperación técnico-financiera a través de agencias, la existencia de foros de integración subregional, la disponibilidad de financiamiento externo, la demanda creciente de datos probatorios por parte de los organismos internacionales y el surgimiento de nuevas metodologías de evaluación en la subregión de América Latina y el Caribe.

Las amenazas encontradas para el desempeño de las FESP en el conjunto de territorios estudiados fueron el crecimiento de la inestabilidad política y la violencia en algunos países y territorios, 
la falta de continuidad en las políticas y los programas de salud por los cambios de gobierno, la politización de las agendas de investigación en salud y la discontinuidad en el flujo de recursos financieros para el sector de la salud debidos a los constantes cambios en las prioridades de la cooperación externa. Otros factores desfavorables para las FESP son la falta de estrategias efectivas que promuevan la participación de los ciudadanos en la toma de decisiones, la ausencia de planeación estratégica acorde con las necesidades de la población, el exceso de burocracia y la insuficiente fiscalización de la gestión de los fondos dirigidos a la salud.

\section{DISCUSIÓN}

Existen divergencias entre las capacidades táctica y estratégica para desempeñar la mayoría de las FESP en el conjunto de organizaciones estudiadas. Esto puede indicar que, en determinadas instituciones, existe una especialización en ciertas competencias y el abandono de otras. Una situación diferente se observa en la FESP 10 (investigación), ya que la mayoría de los países presentaron capacidades tácticas específicas (entre 4,0 y 6,0 puntos), mientras que el desempeño estratégico subregional es bajo (1,6 puntos). Esto puede ser el reflejo de una reducida capacidad, tanto para atender competencias específicas como para cubrir la gama de las mismas. Mientras, puede haber oportunidades para fortalecer algunas de las competencias en la FESP 1 (monitoreo, evaluación y análisis) a partir de la colaboración subregional y en la FESP 10 (investigación) se requiere de apoyo externo. Esta apreciación coincide con otra evaluación realizada por la OPS (6) y refuerza la necesidad de fortalecer la investigación en salud pública en la Región de las Américas.

Ciertas FESP presentan una moderada capacidad estratégica a escala local en un contexto de muy baja capacidad subregional. Así, la FESP 2.3 (laboratorios) muestra un mayor desempeño en Panamá, aunque es solamente de 3,4 puntos, y la FESP 11 (reducción del efecto negativo de emergencias y desastres) presenta un desempeño estratégico mayor en Honduras (4,3 puntos), lo que sugiere la conveniencia de promover una complementación a nivel subregional. Se requiere mayor investigación para identificar los países con posibilidades de colaboración en determinadas FESP.

Las prioridades identificadas en el taller de planeación estratégica refuerzan en general los resultados expuestos sobre el desempeño de las FESP. No obstante, la FESP 8 (desarrollo y capacitación de recursos humanos) fue evaluada con una puntuación promedio de 2,6 puntos, es decir, la gestión de los recursos humanos fue caracterizada como una debilidad general de los países y estados mexicanos evaluados. En este sentido, se resaltó la importancia del fortalecimiento de la gestión de los recursos humanos en salud.

La limitación más importante del presente estudio es la relativamente baja tasa de respuesta a la encuesta en línea por parte de las organizaciones invitadas al estudio que no pertenecen a los ministerios de salud (51,5\%). Sin embargo, este valor se corresponde con lo informado en otras encuestas similares realizadas en la Región de las Américas y fue superior a la de otros estudios nacionales (11-15). Con la excepción de Costa Rica, donde la inclusión de instituciones públicas se consideró insatisfactoria, los expertos de los ministerios de salud de cada país validaron que se hubieran incluido las principales instituciones y que se observaba el balance entre las públicas y las privadas, así como entre las de servicios y las académicas. En el caso costarricense, los hallazgos mostrados - particularmente los relacionados con el desempeño estratégico- deben tomarse con especial cuidado. Los resultados de este trabajo, en general, no constituyen una evaluación en sí y solamente deben utilizarse como elementos para la discusión y elaboración de estrategias de fortalecimiento.

\section{Conclusiones y recomendaciones}

Se requiere mejorar el desempeño estratégico en la mayor parte de las FESP de los territorios analizados. En particular, es necesario reforzar la infraestructura, el equipamiento y los recursos humanos para lograr un mejor desempeño, tanto a nivel táctico como estratégico, a fin de enfrentar los retos en materia de salud pública.
Se recomienda:

- poner en marcha mecanismos de cooperación técnica interinstitucional e intersectorial en cada país y la subregión

- desarrollar un plan consensuado para coordinar la red de instituciones abocadas a realizar acciones de salud pública en cada país y la subregión

- establecer las FESP como prioridad nacional en la captación de la cooperación externa

- reforzar la investigación en salud pública en el ámbito subregional mediante alianzas de los países que integran el Sistema Mesoamericano de Salud Pública.

El Sistema Mesoamericano de Salud Pública, con los recursos financieros de que dispone, tiene la oportunidad de incidir en el fortalecimiento de las FESP a partir de las conclusiones y recomendaciones del presente estudio.

Agradecimientos. Los autores agradecen a la Asociación Internacional de Institutos Nacionales de Salud por el apoyo financiero para la realización del estudio. Agradecen además a Patricia Allen, Marlene Roselló, Jacqueline Peraza y Germana Sánchez, del Instituto Costarricense de Investigación y Enseñanza en Nutrición y Salud (INCIENSA), Costa Rica; Néstor Sosa Montalván, del Instituto Conmemorativo Gorgas de Estudios de la Salud, Panamá; Reynaldo Martorell, de la Universidad de Emory, Estados Unidos de América; y a María Beatriz Duarte y Javier Idrovo, del Instituto Nacional de Salud Pública, México, por el apoyo técnico brindado a esta investigación. De manera particular, se agradece a las autoridades gubernamentales y a los representantes de las organizaciones de la sociedad civil y las instituciones académicas y del sector privado que respondieron la encuesta en línea o la entrevista. Gracias también a los expertos que validaron los resultados del estudio, aportaron información relevante y participaron activamente en el ejercicio de planificación estratégica llevado a cabo en San José, Costa Rica, los días 19 y 20 de julio de 2009.

Conflictos de intereses. Los autores no tienen conflictos de interés que declarar. 


\section{REFERENCIAS}

1. México, Secretaría de Relaciones Exteriores. Proyecto Integración y Desarrollo Mesoamérica. México, D.F.: SRE. Disponible en: http://www.proyectomesoamerica.org/ Acceso el 20 de diciembre de 2012.

2. Iniciativa Mesoamericana de Comercio, Integración y Desarrollo Sostenible [sitio en Internet]. Disponible en: http://www.ini ciativacid.com/ Acceso el 20 de diciembre de 2012.

3. González Block MA, Molina Rodríguez JF, Becerril V, Vargas E, Consejo Directivo del IMSP. Instituto Mesoamericano de Salud Pública: órgano de apoyo técnico y fortalecimiento del Sistema Mesoamericano de Salud Pública. Salud Publica Mex. 2011;53(supl 3):S358-67.

4. Banco de Desarrollo de América Latina. Iniciativa Mesoamericana de Integración y Desarrollo en Mesoamérica [sitio en Internet]. Disponible en: http://www.caf.com/view/ index.asp?ms=19\&pageMs=61643 Acceso el 20 de diciembre de 2012.

5. Organización Panamericana de la Salud. La salud pública en las Américas. Nuevos conceptos, análisis del desempeño y bases para la acción. Washington, D.C.: OPS; 2002. (Publicación Científica y Técnica No. 589.)

6 Organización Panamericana de la Salud. Capacidades en salud pública en América Latina y el Caribe: evaluación y fortalecimiento. Washington, D.C: OPS; 2007.
7. Muñoz F, López-Acuña D, Halverson $P$, Guerra de Macedo C, Hanna W, Larrieu M, et al. Las funciones esenciales de la salud pública: un tema emergente en las reformas del sector de la salud. Rev Panam Salud Publica. 2000;8(1-2):126-34.

8. Asociación Internacional de Institutos Nacionales de Salud Pública. Marco de referencia para la creación y el desarrollo de institutos nacionales de salud pública. Atlanta, GA: IANPHI. Disponible en: http://www. ianphi.org/uploads/file/Framework Español.pdf Acceso el 20 de diciembre de 2012.

9. Binder S, Adigun L, Dusenbury C, Greenspan A, Tanhuanpä P. National public health institutes: contributing to the public good. J Public Health Policy. 2008;29:3-21.

10. México, Instituto Nacional de Salud Pública. Encuesta autoaplicable para las organizaciones. Análisis situacional de organizaciones relacionadas con la salud pública: una propuesta para Mesoamérica-República Dominicana. Disponible en: http://www.insp. $\mathrm{mx} /$ Portal/Centros/ciss/nphi/nphi.php Acceso el 20 de diciembre de 2012.

11. Banco Mundial. Gasto en investigación y desarrollo (\% del PIB). Washington, D.C.: Banco Mundial; sin fecha. Disponible en: http: / / datos.bancomundial.org/indica dor/GB.XPD.RSDV.GD.ZS Acceso el 20 de abril de 2012.
12. Lozar Manfreda K, Bosnjak M, Berzelak J, Haas I, Vehovar V. Web surveys versus other survey modes: a meta-analysis comparing response rates. Int J Mark Res. 2008;50(1):79-104.

13. Cook J, Dickinson HO, Eccles MP. Response rates in postal surveys of healthcare professionals between 1996 and 2005: an observational study. BMC Health Serv Res. 2009;9:160. Disponible en: http://www.biomedcentral. com/content/pdf/1472-6963-9-160.pdf Acceso el 14 de octubre de 2010.

14. Braithwaite D, Emery J, de Lusignan S, Sutton $\mathrm{S}$. Using the Internet to conduct surveys of health professionals: a valid alternative? Fam Pract. 2003;20:545-51.

15. McKinley T, Rogers R, Maclean R. Collecting data from physicians via Web-based surveys: recommendations for improving response rates. Internet J Med Informatics. 2003;1(1). Disponible en: http://www.ispub.com/journal/ the-internet-journal-of-medical-informatics/ volume-1-number-1/ collecting-data-fromphysicians-via-web-based-surveys-recom mendations-for-improving-response-rates. html\#sthash.IcKysH1Z.dpbs Acceso el 20 de diciembre de 2012.

Manuscrito recibido el 11 de noviembre de 2011. Aceptado para publicación, tras revisión, el 29 de noviembre de 2012.

ABSTRACT Objective. Characterize the capacity of public and private institutions in the Central American countries, the Dominican Republic, and the Mexican states of Chiapas and Quintana Roo to perform essential public health functions (EPHFs).

Diagnosis of capacity to perform essential public health functions in the Central American countries, the Dominican Republic, and the Mexican states of Chiapas and Quintana Roo

Key words
Methods. An online survey of 83 organizations in Belize, Costa Rica, the Dominican Republic, El Salvador, Guatemala, Honduras, Nicaragua, Panama, and the Mexican states of Chiapas and Quintana Roo was conducted to learn about their capacity to perform each of the 11 EPHFs. The results were validated in a workshop with representatives of the ministries of health from the seven countries and the two participating Mexican states.

Results. High levels of performance capacity were found most often for EPHF 1 (monitoring, evaluation, and analysis of health status of the population), EPHF 2.1.1 (surveillance, research, and control of risks and threats to public health from infectious diseases), and EPHF 5 (policy development and health planning). The greatest weakness was found in EPHF 2.1.2 (surveillance, research, and monitoring of noninfectious diseases). Asymmetries in EPHF performance within each country mainly revealed weaknesses in the laboratory and public health research functions.

Conclusions. In the countries and territories analyzed, there is a need to improve strategic performance in most of the EPHFs, as well as to strengthen infrastructure, upgrade equipment, and further develop human resources at both the strategic and the tactical levels. A regional approach should be used to take advantage of the different levels of capacity, with a view to greater strengthening and enhanced technical support and cooperation.

Health systems; essential public health functions; Central America; Mexico; Dominican Republic. 